\title{
Meta-analysis: COVID-19 diagnosis in chest CT-master key for radiologists
}

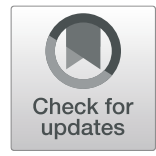

\author{
Soheil Hassanipour ${ }^{1}$, Omid Azadbakht ${ }^{2^{*}}$ (D) Zari Dehnavi ${ }^{3}$, Mohsen Shafiee ${ }^{4}$, Ahmad Badeenezhad ${ }^{5}$, \\ Hossein-Ali Nikbakht ${ }^{6}$, Parsa Faghani Scandarkolaei ${ }^{7}$ and Hassan Bostan ${ }^{4}$
}

\begin{abstract}
Background: COVID-19 was discovered in February in China. Due to the high prevalence of the disease, early detection and rapid isolation of patients are the vital points for controlling the outbreak. The purpose of this study was to determine the correct location of chest CT scan in the diagnosis of COVID-19.

Main text: The current study is a systematic review and meta-analysis. 2959 papers were found in all national and international databases. The study has been reported based on the PRISMA checklist. All analyses were done by CMA Ver. 2 software. The statistical analysis results show that the GGO observation level in the available shape was $46 \%$ in CT scan results, and the consolidation observation level in the general form was 33\% in CT scan results. Pleural effusion was 7\%, and linear opacity observation level was $24 \%$ in CT scan results in the general form. The CT scan test sensitivity level was gained $94.7 \%$, and PCR test sensitivity level was achieved as $94.8 \%$. This level was $89 \%$ in the early stage.

Conclusion: The chest CT has about 24\% higher diagnostic sensitivity than the PCR test, in the early stage. GGO revealed a declining process and also indicates that GGO is an early symptom of the disease in CT scan. Linear opacity is the reason behind the initial dyspnea in coronavirus suffering patients referring to the medical centers. The extra-pulmonary lesions increase in the last stage of the disease that makes the patient's worse.
\end{abstract}

Keywords: Chest CT, Coronavirus, RT-PCR, SARS-CoV-2

\section{Background}

The large family of coronaviruses was first discovered in 1960. Currently, seven species of this family are capable of human-to-human transmission, three of which are lethal. Between 2002 and 2003, the SARS-CoV global epidemic (SARS-CoV) caused 8422 cases and killed 916 people [1]. Another strain, MERS-CoV, was first discovered in Saudi Arabia in September 2012 and eventually killed 858 people in the Middle East [2]. In December 2019, there were reports of a specific type of pneumonia in Wuhan, China. The first patients probably worked or attended one of the seafood markets in the city [3]. Since then, cases have been overgrowing, and the disease has

\footnotetext{
* Correspondence: omidazadbakht68@gmail.com

${ }^{2}$ Department of Radiology Technology, Behbahan Faculty of Medical Sciences, Behbahan, Iran

Full list of author information is available at the end of the article
}

spread to China and beyond. On February 11, 2020, the World Health Organization (WHO) announced that the official name was COVID-19 [4]. The disease primarily causes respiratory tract infection and is transmitted through respiratory droplets and contact. The incubation period is between 1 and 14 days, and the main symptoms are fever, dry cough, and fatigue; besides, nonspecific symptoms include shortness of breath, headache, and muscle pain [5]. Due to the high prevalence of the disease, early detection and rapid isolation of patients are the vital points for controlling the outbreak. Although RNA virus detection is a standard method, it still has its drawbacks and limitations. The first thing about using lab kits is that it takes a day or more to determine the test result. Also, this method can only determine the presence or absence of the disease and not judge the rate of its progression. Imaging modalities are not only useful 
in determining the exact location of lesions but can also help assess the extent of changes. Another is about individual people (especially medical personnel who are directly exposed to the disease); these people require regular tests and perhaps daily tests because of frequent contact. This is not possible because there is a lack of kits in the world. The next reason is the diagnostic quality of these kits. RT-PCR tests only use samples of the upper chest cavity, although in most cases, the lower chest abnormalities occur [6]. It should be noted that in addition to the above reasons, numerous studies have shown that RT-PCR kits are not highly sensitive, and in some cases give false-negative results [7-11]. Early radiological diagnosis can accelerate the planning for conservative care. Computed tomography reports of the first case of COVID-19 (a 47 years old patient) showed that the disease manifestation usually occurs in highdensity, scattered shadows, mainly in the border regions of the lung. Radiology and especially computed tomography $(\mathrm{CT})$ plays an essential role in the early detection, and control of this disorder. Therefore, the purpose of this study was to determine the correct location of imaging modalities in the diagnosis of COVID-19.

\section{Main text}

\section{Methods}

The current study is a systematic review and meta-analysis about COVID-19 diagnosis in chest CT: master key for radiologists. The study was designed and implemented in 2020. This study has been reported based on the PRISMA (Preferred Reporting Items for Systematic Reviews and Meta-Analysis) checklist.

\section{Search strategy}

On March 17, 2020, the present study conducting researchers got to search six international databases, including MEDLINE/PubMed, ProQuest, Scopus, ISI, Embase, Cochrane library, and Google Scholar as the Gray Literature.

The selected keywords for the international databases were coronavirus, coronavirus, 2019 nCov, COVID-19, COVID 19, 2019-nCov, novel coronavirus, novel coronavirus 2019, nCov, severe acute respiratory syndrome coronavirus, Wuhan coronavirus, Wuhan seafood market pneumonia virus, and SARS-nCov2.

The collected data were entered into EndNote version X7 software, and the repeated papers were automatically removed. It is worth noting that the two researchers examined the documents separately.

\section{Inclusion and exclusion criteria of related studies}

All studies conducted until March 17, 2020, discovered with the keywords in six databases, were included in the study. Of course, the studies whose full version was not at hand, the non-English ones, and the ones not dealing with the current topic were crossed out.

\section{Quality assessment}

To analyze, and control the papers' quality, the JBI checklist developed by Joanna Briggs Institute was applied, and it is used to evaluate various types of studies qualitatively. In this research, three types of lists (cross-sectional, case series, and cohort) were picked base on the inclusion study type. Each list contains different questions that are generally based on the quality of each study, as including it or not, in the final analysis, is decided.

\section{Screening studies}

The initial search of the studies was done by two individuals (S.H, and HA.N). The screening of the studies, extracting the results, and also evaluating the papers' quality was performed by two persons (Z.D and P.F) separately. In case of a lack of consensus, the team supervisor (O.A) announced the final comment about that paper.

\section{Studies' related bias risk}

Sub-group analysis and Egger tests have been employed to analyze the probability of bias in included studies.

\section{Statistical analysis}

The heterogeneity among the studies has been checked by the Cochran test (significance level $<0.1$ ), and its combination using $I^{2}$ statistics (significance level $>50 \%$ ). In the heterogeneity existence, the random-effects model was applied by the variance inversion method, and in the case of no heterogeneity, the fixed-effects model was employed.

For analyzing the data, there were seven states as GGO, consolidation, GGO, and consolidation; CP pattern; lymphadenopathy; pleural effusion; and linear opacity having the highest report in the studies were chosen. Then, the probability of observing the onset of symptoms, each of the states was analyzed based on the disease's stage (early, progress, and advance).

To measure the two tests' sensitivity, two tests as CT scan, and PRC were first separately employed based on the disease stage. After that, the comparison between the two trials was analyzed based on the calculated OR (odds ratio) index.

The method used in the present research, known as the calculated odds ratio (OR) index, was applied so that the readers better figure out the paper. Comprehensive Meta-Analysis (CMA) software owns the potential to combine various indices and to integrate the sample size, and the index difference is compared. According to the conclusion, the OR index can well illustrate the gap existing between the two tests. All analyses were done by CMA Ver. 2 software. 


\section{Results}

\section{Studies search description}

Two thousand nine hundred fifty-nine papers were found in all national and international databases that, after deleting the repeated ones, 1482 articles got into the analysis stage in terms of the title and abstract. After addressing the papers' titles and abstracts, 68 ones got into the next step, where the full paper's text was surveyed, and 21 reports reached the final step. It is worth stating that the included papers' references were investigated to add the relevant articles. In the studies' screening stages, the reviews were excluded from analysis due to various reasons encompassing irrelevant topic, irrelevant study population, and repeated results. The included studies' flow-chart is depicted in Fig. 1.

\section{Included study description}

The studies' demographics are listed in Table 1. Out of 21 included studies, 17 cases were of China, two studies in Italy, and two studies in South Korea.

\section{Results of quality assessment}

The results obtained from analyzing the studies' qualitative control based on the included reviews indicate that all reviews possess desirable quality. The total results of the studies' qualitative assessment are covered in supplementary file 1 .

\section{Heterogeneity-related results}

Heterogeneity investigation-derived results of all analyses have been shown that in Table 2 .

\section{Results of meta-analysis}

GGO

As the results suggest, the GGO observation level in the general form was $46 \%$ (95\% confidence intervals [CI]; $38-54)$ in CT scan results. This level was $69 \%$ (95\% CI; $56-79)$ in the early stage, $65 \%(95 \% \mathrm{CI} ; 22-92)$ in the progress stage, and $32 \%(95 \% \mathrm{CI} ; 23-41)$ in the advance stage. The GGO observation findings related to total results in the patients' CT scan are seen in Fig. 2.

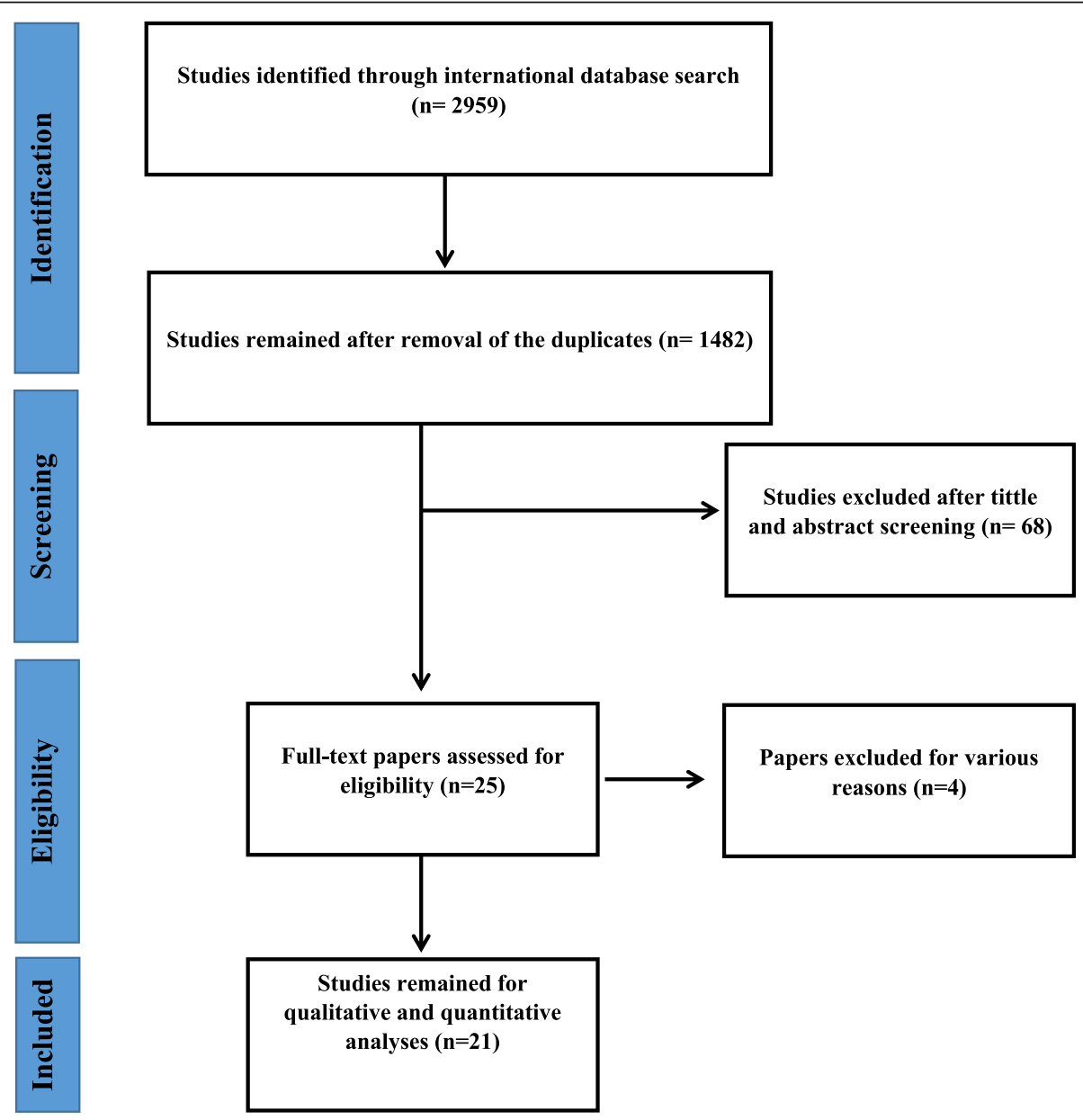

Fig. 1 Flowchart of the included eligible studies in a systematic review 


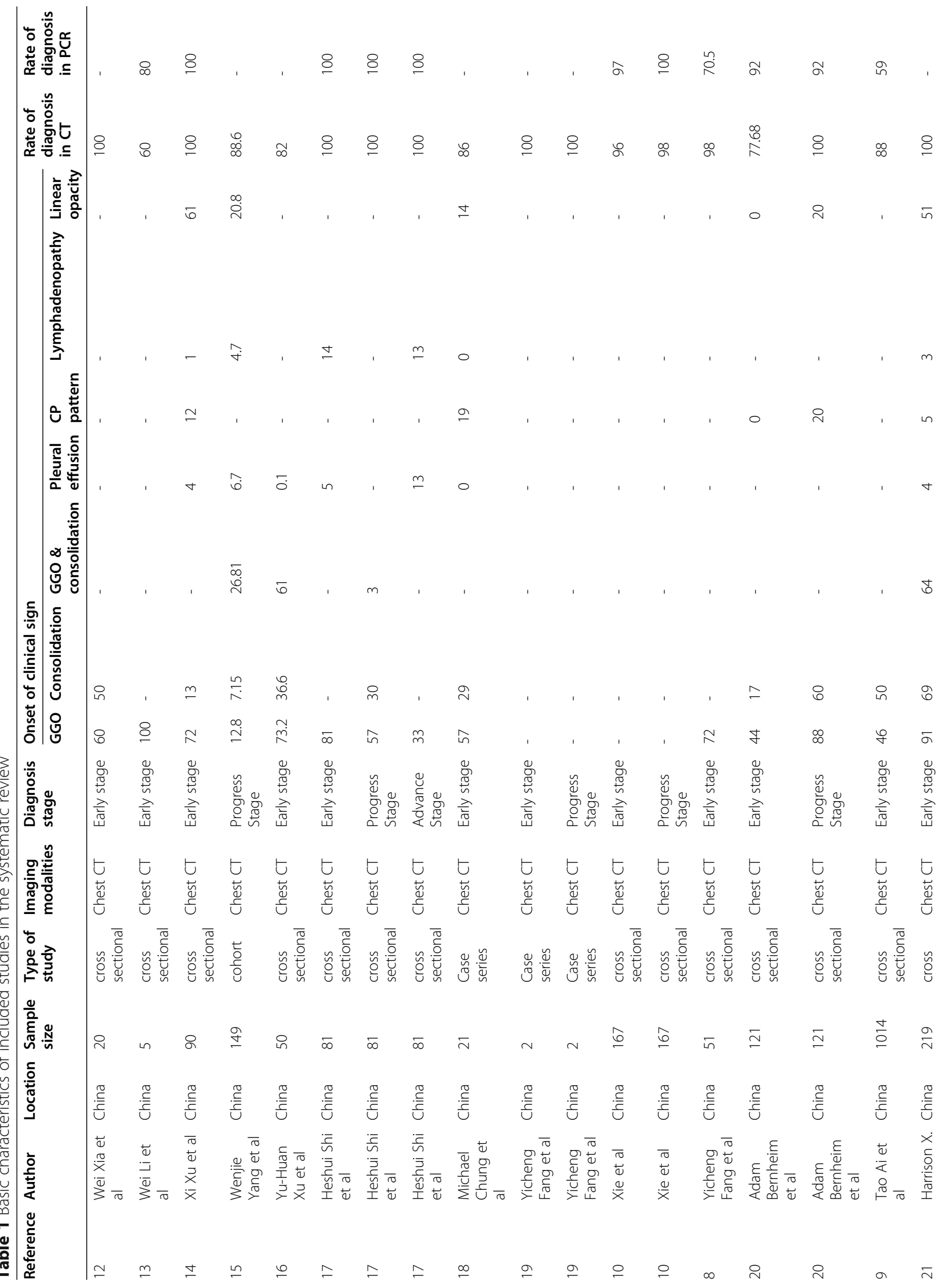




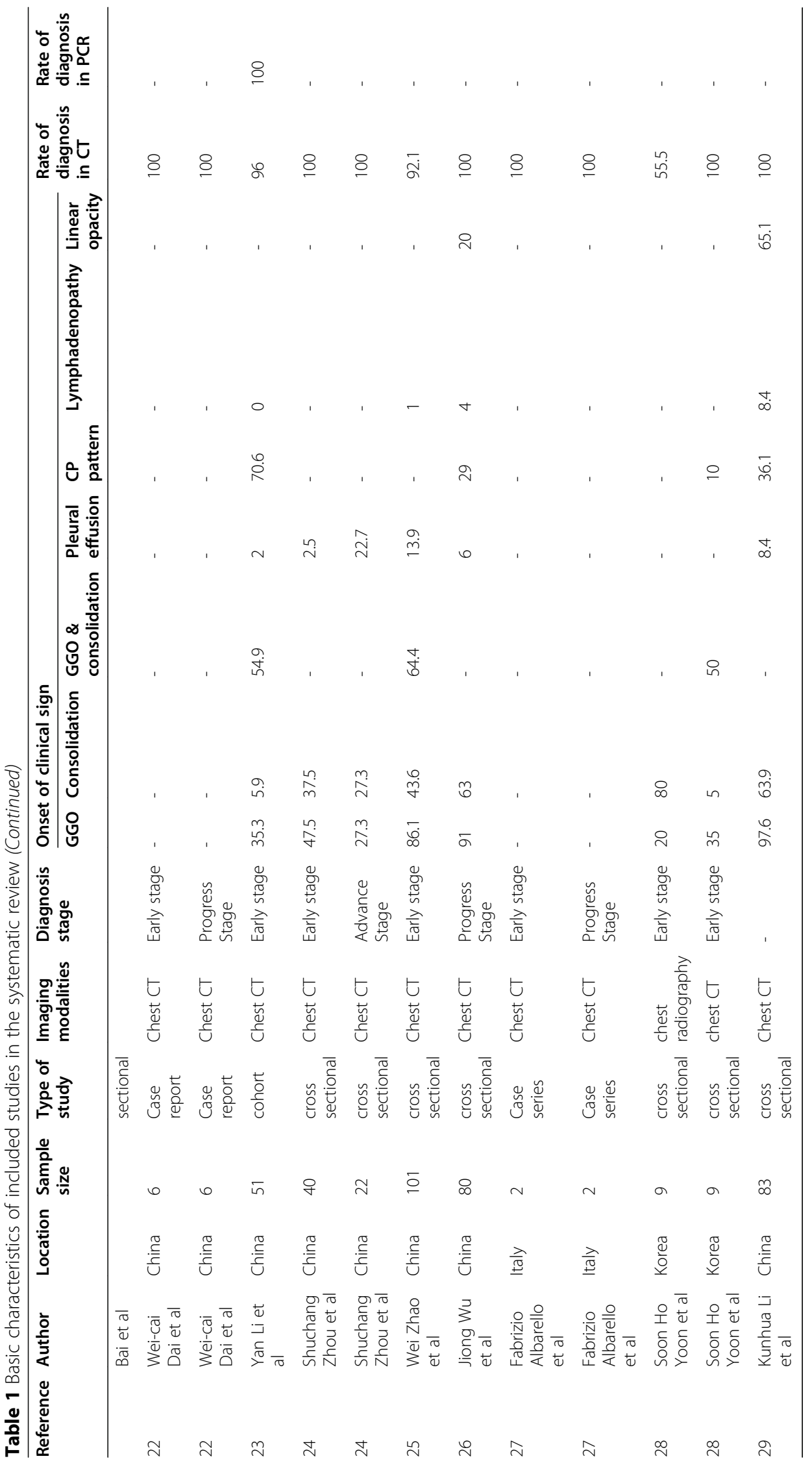


Table 2 Results of heterogeneity among included studies

\begin{tabular}{|c|c|c|c|c|c|c|c|}
\hline Variable & Category & \# of studies & Q-value & Df (Q) & I-squared & $P$-value & Selected model \\
\hline \multirow[t]{4}{*}{ GGO } & Advance stage & 2 & 0.291 & 1 & 0.0 & 0.590 & Fixed \\
\hline & Early stage & 15 & 231.07 & 14 & 93.94 & $<0.001$ & Random \\
\hline & Progress stage & 4 & 144.53 & 3 & 97.92 & $<0.001$ & Random \\
\hline & Overall & 21 & 394.99 & 20 & 94.93 & $<0.001$ & Random \\
\hline \multirow[t]{4}{*}{ Consolidation } & Advance stage & 1 & 0.0 & 0 & 0.0 & 0.999 & Fixed \\
\hline & Early stage & 12 & 151.16 & 11 & 92.72 & $<0.001$ & Random \\
\hline & Progress stage & 4 & 81.91 & 3 & 96.33 & $<0.001$ & Random \\
\hline & Overall & 17 & 238.65 & 16 & 93.29 & $<0.001$ & Random \\
\hline \multirow[t]{3}{*}{ GGO \& Consolidation } & Early stage & 5 & 2.65 & 4 & 0.0 & 0.618 & Fixed \\
\hline & Progress stage & 2 & 13.07 & 1 & 92.35 & $<0.001$ & Random \\
\hline & Overall & 7 & 81.49 & 6 & 92.63 & $<0.001$ & Random \\
\hline \multirow[t]{3}{*}{ CP Pattern } & Early stage & 5 & 2.65 & 4 & 0.0 & 0.618 & Fixed \\
\hline & Progress stage & 2 & 13.07 & 1 & 92.35 & $<0.001$ & Random \\
\hline & Overall & 7 & 81.49 & 6 & 92.63 & $<0.001$ & Random \\
\hline \multirow[t]{4}{*}{ Lymphadenopathy } & Advance stage & 2 & 1.45 & 1 & 31.21 & 0.228 & Fixed \\
\hline & Early stage & 6 & 18.56 & 5 & 73.06 & 0.002 & Random \\
\hline & Progress stage & 2 & 0.11 & 1 & 0.0 & 0.738 & Fixed \\
\hline & Overall & 10 & 31.51 & 9 & 71.44 & $<0.001$ & Random \\
\hline \multirow[t]{4}{*}{ Pleural effusion } & Advance stage & 1 & 0.0 & 0 & 0.0 & 0.999 & Fixed \\
\hline & Early stage & 7 & 14.76 & 6 & 59.34 & 0.022 & Random \\
\hline & Progress stage & 2 & 0.01 & 1 & 0.0 & 0.893 & Fixed \\
\hline & Overall & 10 & 17.98 & 9 & 50.95 & 0.035 & Random \\
\hline \multirow[t]{3}{*}{ Linear Opacity } & Early stage & 4 & 16.53 & 3 & 81.85 & 0.001 & Random \\
\hline & Progress stage & 3 & 0.04 & 2 & 0.0 & 0.978 & Fixed \\
\hline & Overall & 7 & 103.26 & 6 & 94.19 & $<0.001$ & Random \\
\hline \multirow[t]{4}{*}{ Diagnosis test (CT scan) } & Advance stage & 2 & 0.40 & 1 & 0.0 & 0.523 & Fixed \\
\hline & Early stage & 21 & 79.62 & 20 & 74.88 & $<0.001$ & Random \\
\hline & Progress stage & 6 & 8.97 & 5 & 44.29 & 0.110 & Fixed \\
\hline & Overall & 29 & 89.0 & 28 & 70.52 & $<0.001$ & Random \\
\hline \multirow[t]{4}{*}{ Diagnosis test (PCR) } & Advance stage & 1 & 0.0 & 0 & 0.0 & 0.999 & Fixed \\
\hline & Early stage & 6 & 34.73 & 5 & 85.60 & $<0.001$ & Random \\
\hline & Progress stage & 5 & 12.14 & 4 & 67.05 & 0.016 & Random \\
\hline & Overall & 12 & 177.27 & 11 & 93.79 & $<0.001$ & Random \\
\hline
\end{tabular}

\section{Consolidation}

The statistical analysis results show that the consolidation observation level in the general form was 33\% (95\% CI; 25-43) in CT scan results. This level was 34\% (95\% CI; $24-46)$ in the early stage, $35 \%$ (95\% CI; $25-43$ ) in the progress stage, and $27 \%$ (95\% CI; $12-48)$ in the advance stage. The total results of the consolidation observation findings in the patients' CT scan are illustrated in Fig. 3.

\section{GGO and consolidation}

According to the results, GGO, and consolidation observation level in the general form was $61 \%$ (95\% CI; 57-66) in CT scan results. This level was 62\% (95\% CI; 57-66) in the early stage and $9 \%(95 \% \mathrm{CI} ; 0-59)$ in the progress stage. The total results of GGO and consolidation observation findings in the patients' CT scan are depicted in Fig. 4.

\section{Crazy paving pattern (CP pattern)}

Based on the results, the CP pattern observation level in the general form was $23 \%(95 \% \mathrm{CI} ; 16-32)$ in CT scan results. This level was $21 \%(95 \% \mathrm{CI} ; 7-47)$ in the early stage and $23 \%(95 \% \mathrm{CI} ; 16-33)$ in the 


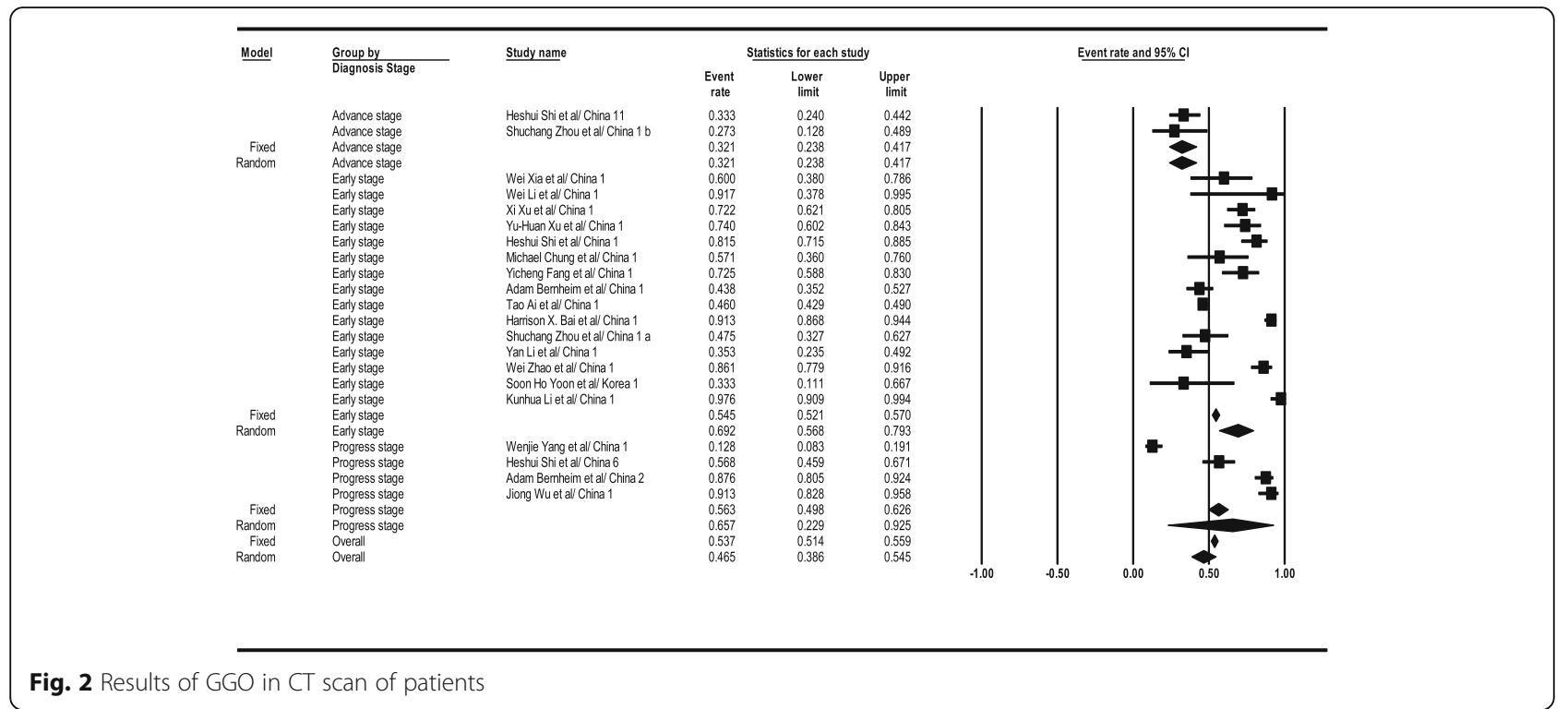

progress stage. CP pattern-related total results in the ST scan of patients are shown in Fig. 5.

\section{Lymphadenopathy}

The statistical analysis extracted results denoted that lymphadenopathy observation level was 6\% (95\% CI; 410) in CT scan results. This level was $4 \%$ (95\% CI; 1-9) in the early stage, $4 \%(95 \% \mathrm{CI} ; 2-8)$ in the progress stage, and $15 \%$ (95\% CI; 9-23) in the advance stage. Lymphadenopathy observation-based total results in the CT scan of patients are shown that in Fig. 6.

\section{Pleural effusion}

The statistical analysis results indicated that pleural effusion in the general form was $7 \%(95 \% \mathrm{CI} ; 5-10)$ in CT scan results. This level was $5 \%(95 \% \mathrm{CI} ; 3-9)$ in the early stage, $6 \%(95 \% \mathrm{CI} ; 4-10)$ in the progress stage, and $12 \%$ (95\% CI; 6-21) in the advance stage. The total results of pleural effusion observation in the patients' CT scans are depicted in Fig. 7.

\section{Linear opacity}

Based on the results, the linear opacity observation level in the general form was $24 \%$ (95\% CI; 20-29) in CT scan

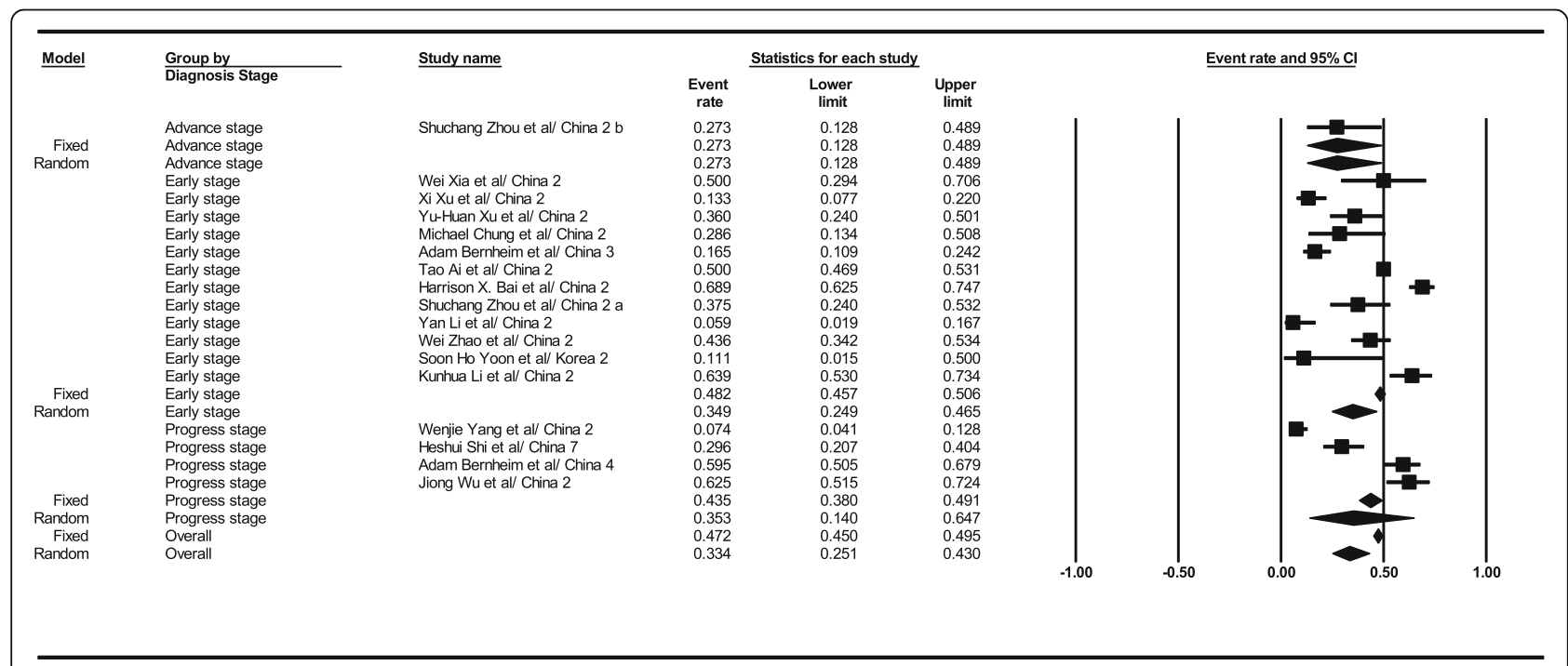

Fig. 3 Results of GGO in CT scan of patients 


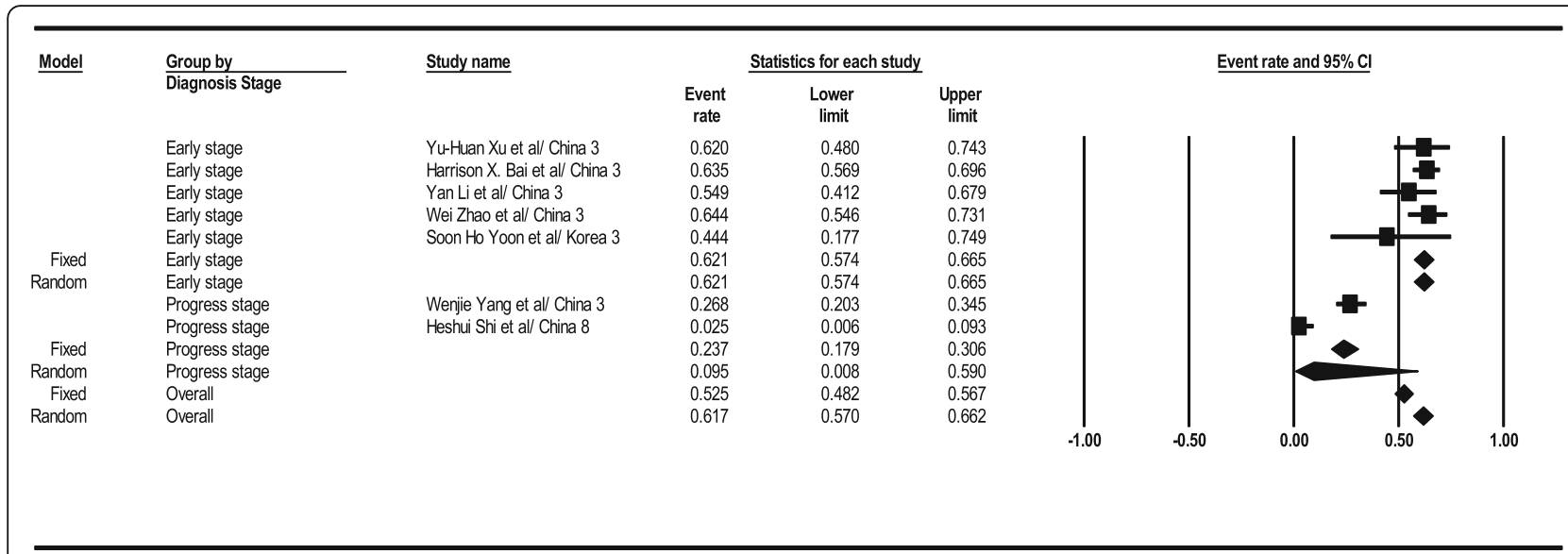

Fig. 4 Results of GGO in CT scan of patients

results. This level was 52\% (95\% CI; 38-65) in the early stage and $20 \%(95 \% \mathrm{CI} ; 16-24)$ in the progress stage. Linear opacity-related total results in the patients' CT scans are demonstrated in Fig. 8.

\section{CT scan test sensitivity}

As the statistical analysis results suggest, CT scan test sensitivity level in the general form was gained $94.7 \%$ (95\% CI; 91.8-96.6). This level was 94.2\% (95\% CI; 90.796.4) in the early stage, $90 \%$ (95\% CI; 85.1-93.6) in the progress stage, and $99 \%(95 \% \mathrm{CI}$; $92.3-99.8)$ in the advance stage. CT scan test sensitivity-related total results are depicted in Fig. 9.

\section{PCR test sensitivity}

According to statistical analysis results, the PCR test sensitivity level was achieved as 94.8\% (95\% CI; 90.4-97.2) in the general form. This level was $89 \%$
(95\% CI; 72.3-96.3) in the early stage, $95.8 \%(95 \%$ $\mathrm{CI}$; 90.8-98.1) in the progress stage, and $99.4 \%(95 \%$ CI; 91-100) in the advance stage. PCR test sensitivity relevant total results are given in Fig. 10.

\section{Comparing $C T$ scan and PCR tests}

As revealed by calculated OR index, generally speaking, CT scan and PCR tests did not show statistically significant $\left(\mathrm{OR}=1.001,95 \% \mathrm{CI} ; 0.91-1.11, p=0.851 ; I^{2}=94.97\right.$, $p<0.001)$, while according to data analysis, CT scan test showed approximately $24 \%$ higher sensitivity than PCR in the early stage (comparison of CT and PCR tests section, based on the data analyzed in Fig. 11), the finding which was not statistically significant $(\mathrm{OR}=1.24,95 \% \mathrm{CI}$; 0.76-2.03, $\left.p=0.383 ; I^{2}=94.97, p<0.001\right)$. The total results of a comparison between CT scan and PCR tests are seen in Fig. 11.

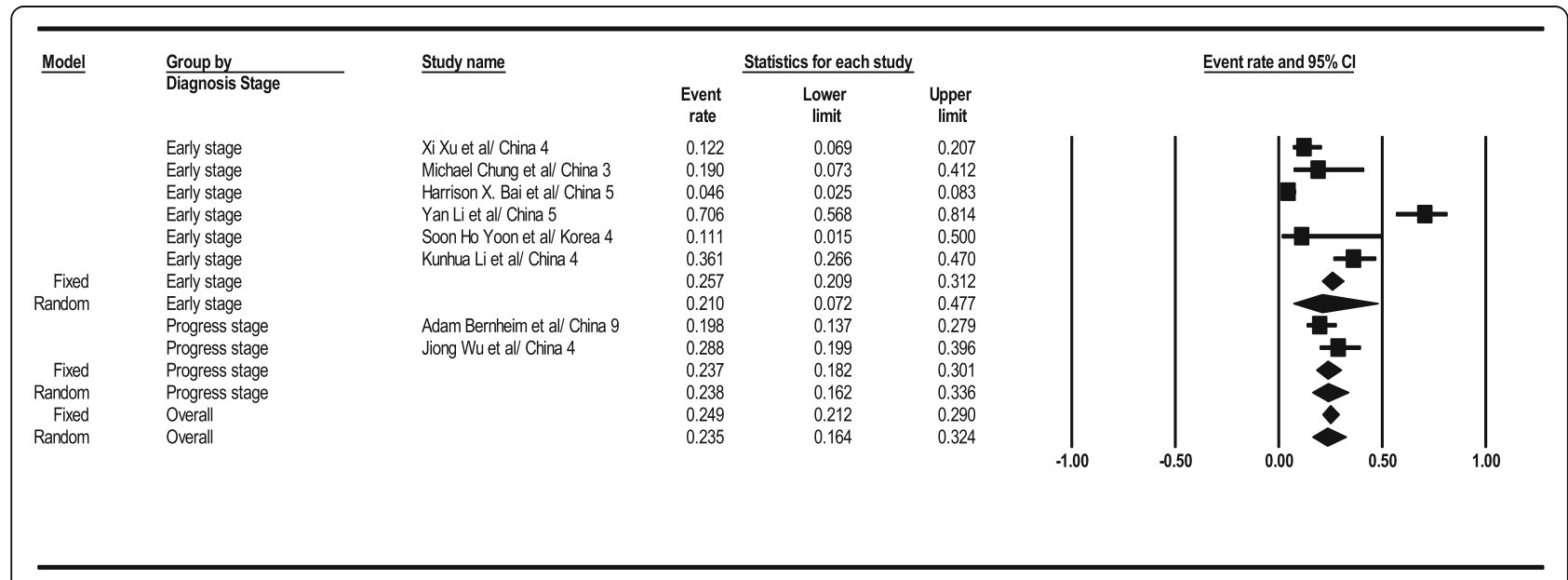

Fig. 5 Results of GGO in CT scan of patients 


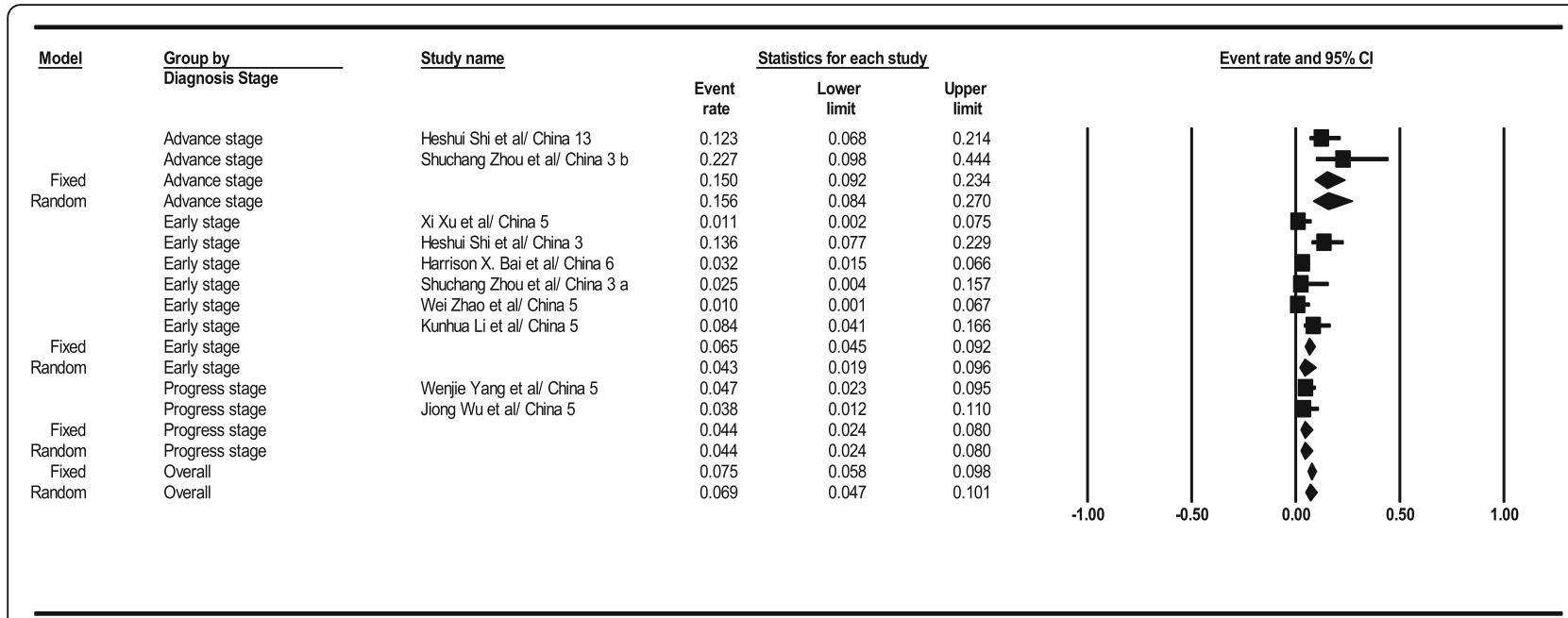

Fig. 6 Results of GGO in CT scan of patients

\section{Publication bias}

To investigate publication bias in the results, the Egger test and funnel plot extracted results were applied. The obtained results indicate no significant error spotted in the works $(p=0.747)$. The funnel plot of publication bias analysis is observed in Fig. 12.

\section{Discussion}

One of the goals the current research pursues is to compare the diagnostic percent of chest CT and RT-PCR to obtain the most effective method for diagnosing COVID-19, and the findings exhibit that chest CT scan was $24 \%$ more sensitive than RT-PCR in the early stages of the disease diagnosis. Likewise, the study done by Tao Ai showed that RT-PCR plays a fundamental role in isolating and hospitalizing COVID-19 suffering patients.
Still, the factors like sampling operation, sampling sources (sampling location in the respiratory tract or lung), sampling time, and the diagnostic kit's quality impair it relative to chest CT in the early disease diagnostic stages. On the one hand, chest CT determines pulmonary engagement and progression of the disease and is less affected by human error [6-11, 30-34].

The present study-derived findings suggest that GGO outbreak level is around 69\% in the early diagnostic stages of COVID-19 in chest CT. Similarly, GGO was seen in the majority of the studies on coronavirus suffering patients undergoing CT scan tests [17, 30-32, 35]. In some studies such as $\mathrm{Li}$ et al.'s research, only GGO was analyzed in chest CT; this issue indicates the physicians' experimental perception of this symptom outbreak in chest CT images [13, 24, 35, 36]. Generally speaking,

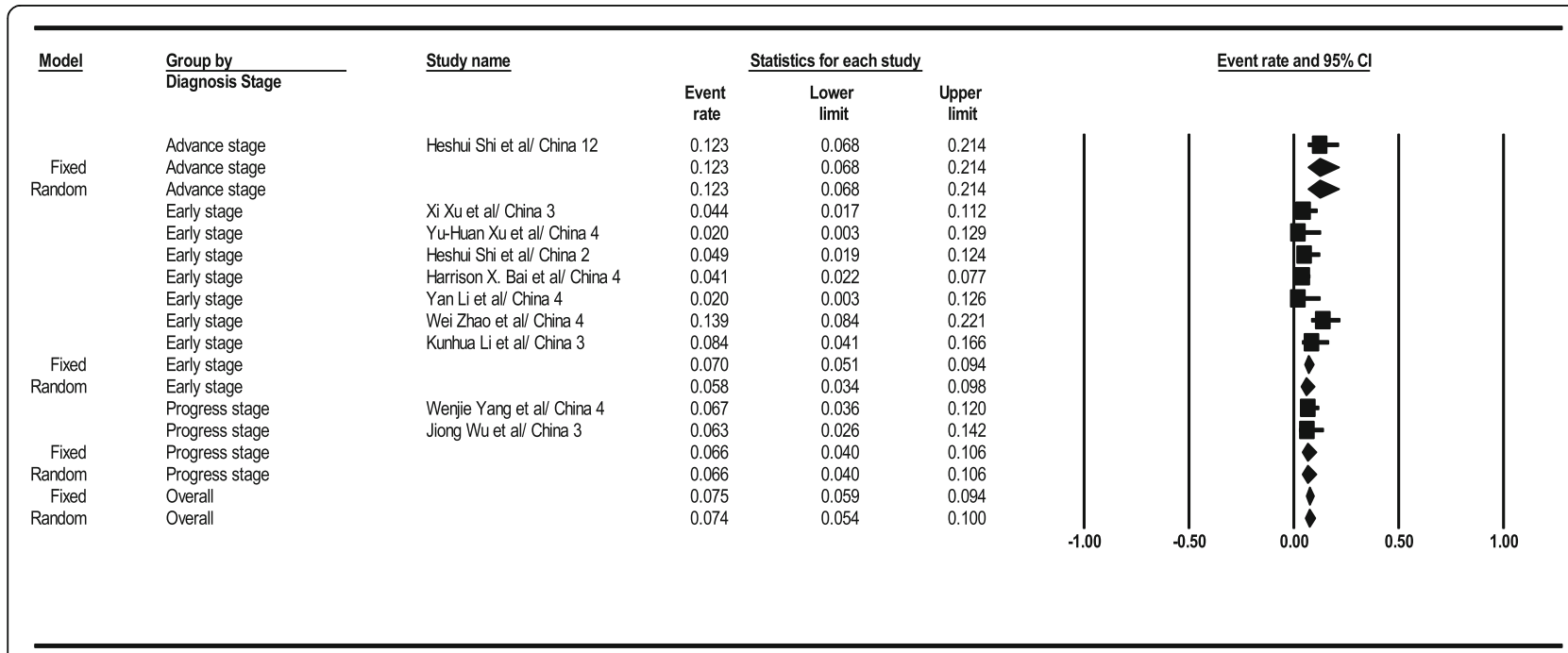

Fig. 7 Results of GGO in CT scan of patients 


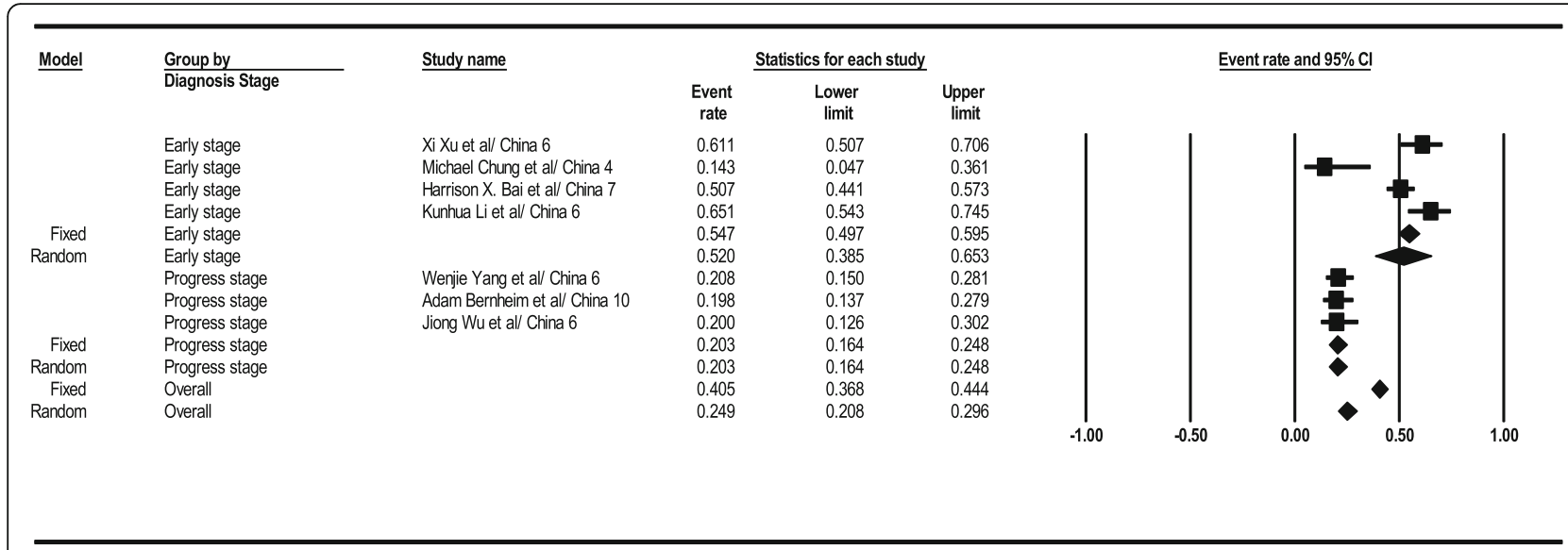

Fig. 8 Results of GGO in CT scan of patients

the abovementioned findings suggest that probably GGO is of coronavirus clinical sign in chest CT images, and this itself approves the present research [37]. It is worth mentioning that in some tasks, such as the one by $\mathrm{Li}$ et al., only the GGO was analyzed in chest CT. This issue indicates the physicians' experimental perception of this symptom outbreak in chest CT images [13, 24, $35,36]$. Generally speaking, the abovementioned findings suggest that probably the GGO is of coronavirus suffering patients' clinical symptoms in chest CT images, and this itself approves the present research [37]. Besides, as stated by the study of $\mathrm{Xu}$ et al., crazy paving pattern is a particular state of the GGO. Thus, it has to include a lower percentage of GGO; the issue shown by this study [35].

The studies' analysis-derived results revealed that consolidation symptom's incidence in chest CT images is generally 33\% (a pulmonary consolidation is a region of normally compressible lung tissue that has filled with liquid instead of air), and also consolidation results from inflammatory exudation by alveoli filling, which means the virus spreads through the respiratory epithelium and

\begin{tabular}{|c|c|c|c|c|c|c|c|c|c|c|}
\hline \multirow[t]{3}{*}{$\underline{\text { Model }}$} & \multirow{2}{*}{$\frac{\text { Group by }}{\text { Diagnosis Stage }}$} & \multirow[t]{2}{*}{$\underline{\text { Study name }}$} & \multicolumn{3}{|c|}{ Statistics for each study } & \multicolumn{5}{|c|}{ Event rate and $95 \% \mathrm{Cl}$} \\
\hline & & & $\begin{array}{c}\text { Event } \\
\text { rate }\end{array}$ & $\begin{array}{l}\text { Lower } \\
\text { limit }\end{array}$ & $\begin{array}{l}\text { Upper } \\
\text { limit }\end{array}$ & & & & & \\
\hline & Advance stage & Heshui Shi et al/ China 14 & 0.994 & 0.910 & 1.000 & & & & & \\
\hline & Advance stage & Shuchang Zhou et al/ China $4 \mathrm{~b}$ & 0.978 & 0.732 & 0.999 & & & & & \\
\hline \multirow{23}{*}{$\begin{array}{r}\begin{array}{r}\text { Fixed } \\
\text { Random }\end{array}\end{array}$} & Advance stage & & 0.989 & 0.923 & 0.998 & & & & & \\
\hline & Advance stage & & 0.989 & 0.923 & 0.998 & & & & & \\
\hline & Early stage & Wei Xia et al/ China 3 & 0.976 & 0.713 & 0.999 & & & & & \\
\hline & Early stage & Wei Li et al/ China 2 & 0.600 & 0.200 & 0.900 & & & & & \\
\hline & Early stage & Xi Xu et al/ China 7 & 0.995 & 0.918 & 1.000 & & & & & \\
\hline & Early stage & Yu-Huan Xu et al/ China 5 & 0.820 & 0.689 & 0.904 & & & & & \\
\hline & Early stage & Heshui Shi et al/ China 4 & 0.994 & 0.910 & 1.000 & & & & & \\
\hline & Early stage & Michael Chung et al/ China 5 & 0.857 & 0.639 & 0.953 & & & & & \\
\hline & Early stage & Yicheng Fang et al/ China 1a & 0.833 & 0.194 & 0.990 & & & & & \\
\hline & Early stage & Xie et al/ China 1 & 0.964 & 0.922 & 0.984 & & & & & \\
\hline & Early stage & Xie et al/ China 3 & 0.982 & 0.946 & 0.994 & & & & & \\
\hline & Early stage & Yicheng Fang et al/ China 2 & 0.980 & 0.874 & 0.997 & & & & & \\
\hline & Early stage & Adam Bernheim et al/ China 5 & 0.777 & 0.694 & 0.842 & & & & & \\
\hline & Early stage & Adam Bernheim et al/ China 7 & 0.996 & 0.938 & 1.000 & & & & & \\
\hline & Early stage & Tao Ai et al/ China 3 & 0.880 & 0.858 & 0.898 & & & & & \\
\hline & Early stage & Harrison X. Bai et al/ China 8 & 0.998 & 0.965 & 1.000 & & & & & \\
\hline & Early stage & Wei-cai Dai et al/ China 1 & 0.929 & 0.423 & 0.996 & & & & & \\
\hline & Early stage & Shuchang Zhou et al/ China 4 a & 0.988 & 0.833 & 0.999 & & & & & \\
\hline & Early stage & Yan Li et al/ China 6 & 0.961 & 0.856 & 0.990 & & & & & \\
\hline & Early stage & Wei Zhao et al/ China 6 & 0.921 & 0.850 & 0.960 & & & & & \\
\hline & Early stage & Fabrizio Albarello et al/ Italy 1 & 0.833 & 0.194 & 0.990 & & & & & \\
\hline & Early stage & Soon Ho Yoon et al/ Korea 5 & 0.950 & 0.525 & 0.997 & & & & & \\
\hline & Early stage & Kunhua Li et al/ China 7 & 0.994 & 0.912 & 1.000 & & & & & \\
\hline Fixed & Early stage & & 0.886 & 0.870 & 0.901 & & & & & \\
\hline \multirow[t]{7}{*}{ Random } & Early stage & & 0.942 & 0.907 & 0.964 & & & & & \\
\hline & Progress stage & Wenjie Yang et al/ China 7 & 0.886 & 0.824 & 0.928 & & & & & \\
\hline & Progress stage & Heshui Shi et al/ China 9 & 0.994 & 0.910 & 1.000 & & & & & \\
\hline & Progress stage & Yicheng Fang et al/ China 2a & 0.833 & 0.194 & 0.990 & & & & & \\
\hline & Progress stage & Wei-cai Dai et al/ China 2 & 0.929 & 0.423 & 0.996 & & & & & \\
\hline & Progress stage & Jiong Wu et al/ China 7 & 0.994 & 0.909 & 1.000 & & & & & \\
\hline & Progress stage & Fabrizio Albarello et al/ Italy 2 & 0.833 & 0.194 & 0.990 & & & & & \\
\hline Fixed & Progress stage & & 0.902 & 0.851 & 0.936 & & & & & \\
\hline Random & Progress stage & & 0.943 & 0.830 & 0.982 & & & & & \\
\hline Fixed & Overall & & 0.889 & 0.874 & 0.903 & & & & & \\
\hline \multirow{2}{*}{ Random } & Overall & & 0.947 & 0.918 & 0.966 & & & & & $\mathbf{V}$ \\
\hline & & & & & & -1.00 & -0.50 & 0.00 & 0.50 & 1.00 \\
\hline
\end{tabular}




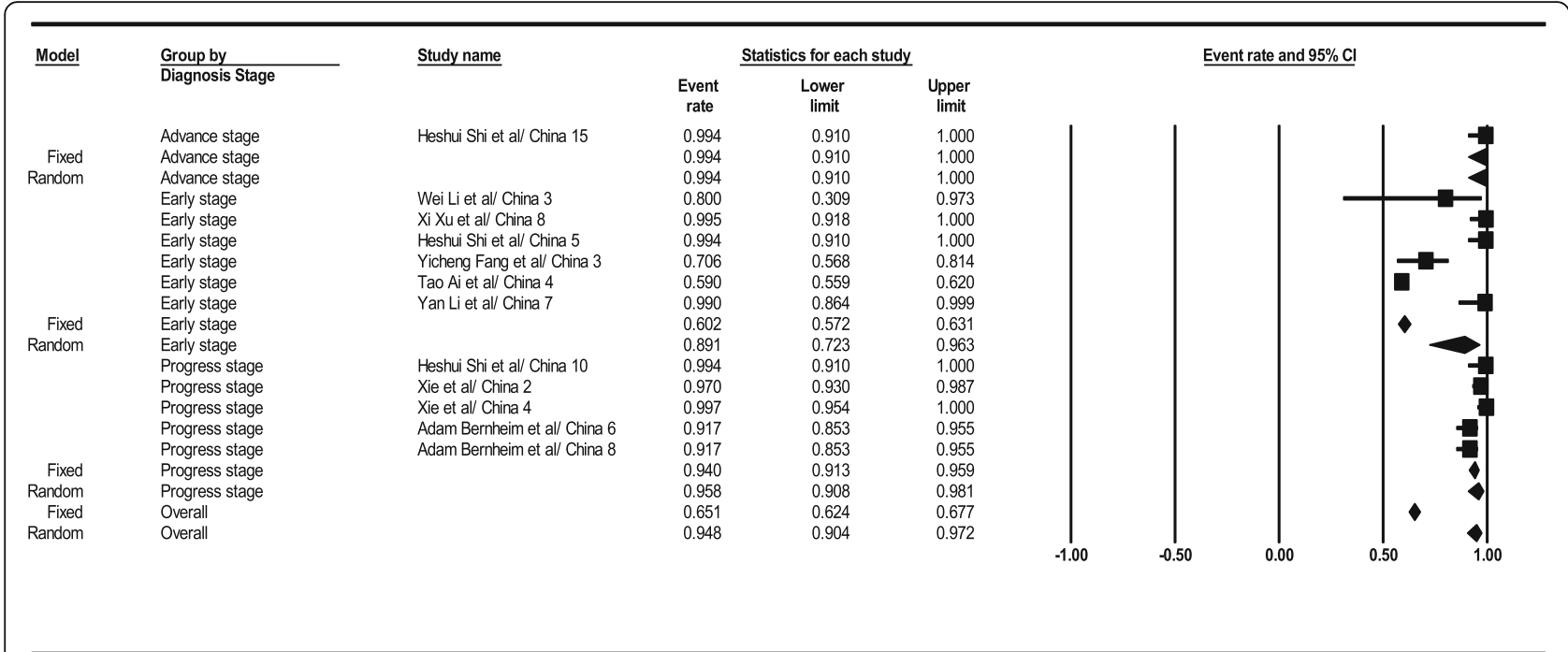

Fig. 10 Results of GGO in CT scan of patients

leads to alveoli damage and necrotic bronchitis. It has to be stated that this finding has been investigated by $\mathrm{Li}$ et al. [29, 38-40]. MIX state or the simultaneous presence of consolidation, and GGO in the early stage of diagnosis has gained a high percentage (61\%). This high percentage has been confirmed in several other studies $[15,21,23,41]$. This study extracted results showing that the linear opacity is generally $24 \%$ and $52 \%$ in the early stage of diagnosis. The linear opacity indicates a decrease of air to tissue ratio due to pathogens. This is probably the reason behind the initial dyspnea in coronavirus suffering patients referring to the medical centers. The presence of the linear opacity in chest CT images of the patients has been supported in the study by Chung et al. [18]. As stated by the research of Kunhua et al., the extra-pulmonary lesions such as pleural effusion signal severe inflammation as less than $10 \%$ in the present study generally and increasing in the last stage of the disease due to the grave condition of some of the patients [29].

Comparing the different stages of the disease diagnosis in this study demonstrates that the clinical manifestations

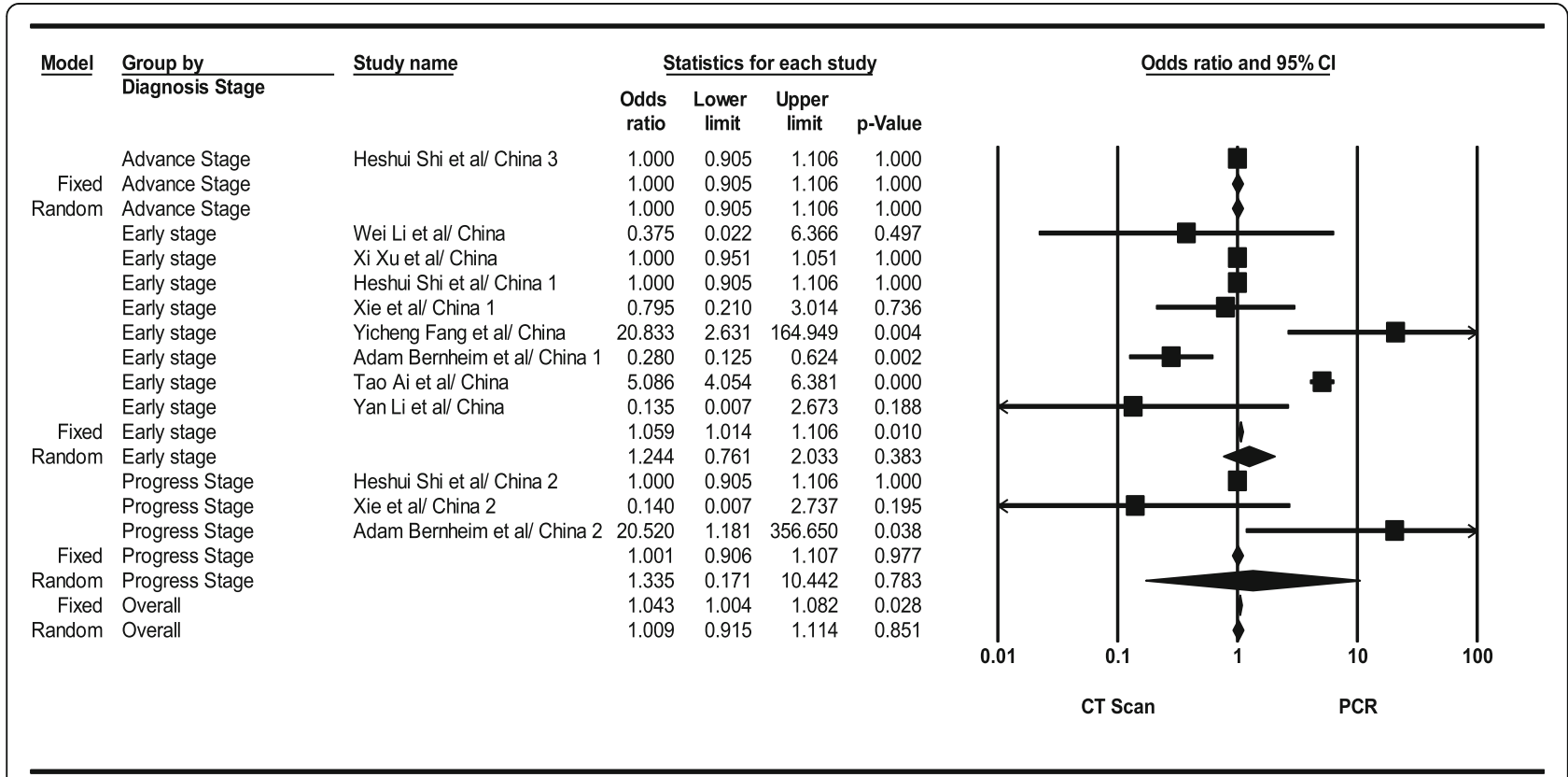

Fig. 11 Results of GGO in CT scan of patients 


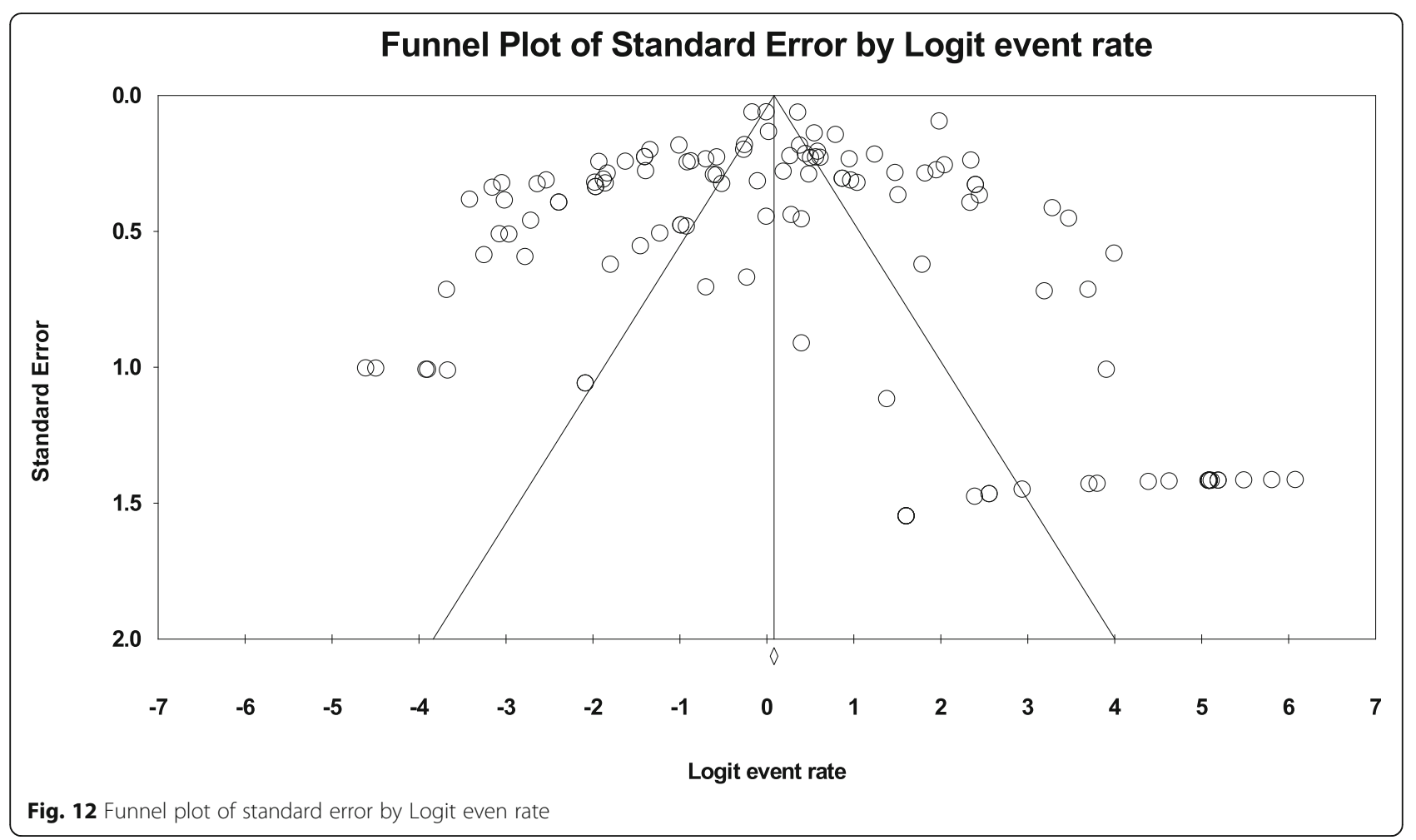

of the study did not change much (symptoms of coronavirus on chest CT imaging). However, the GGO revealed a declining process, and it is probably due to recovery over time and also indicates that the GGO is an early symptom of the disease $[24,42]$.

\section{Conclusion}

To sum up, the chest CT and PCR-induced results are almost identical with no meaningful difference. Though, in the early stage of the disease diagnosis, chest CT has about 24\% higher diagnostic accuracy than PCR test. In the early stage of disease diagnosis as the most critical one, the most prevalent COVID-19-induced symptoms in chest CT are GGO and Mix (GGO and consolidation). Analyzing all stages of the disease diagnosis showed that the highest incidence in chest CT images are related to GGO and Mix (GGO, and Consolidation), and the least are of pleural effusion and lymphadenopathy. Moreover, in the current research, it has been found that GGO is the early symptom of the disease and declines over time. Also, the extrapulmonary lesions such as pleural effusion signal severe inflammation in the disease advance stage due to the grave condition of some of the patients have increased.

\section{Abbreviations}

CT: Computer tomography; CP Pattern: Crazy-paving pattern; PCR: Polymerase chain reaction; RT-PCR: Reverse transcription-polymerase chain reaction

\section{Supplementary Information}

The online version contains supplementary material available at https://doi, org/10.1186/s43055-021-00457-6.

Additional file 1.

Acknowledgements

This study has been supported by the Behbahan Faculty of Medical Science.

Authors' contributions

$\mathrm{SH}, \mathrm{O} \mathrm{A}, \mathrm{ZD}, \mathrm{HA} \mathrm{N}, \mathrm{M} \mathrm{SH}, \mathrm{A}$ B, and P A conceived and planned the experiments. $\mathrm{O} A, S \mathrm{H}, \mathrm{M} \mathrm{SH}$, and Z D carried out the experiments. $\mathrm{O} A, \mathrm{~S} \mathrm{H}$, and M SH planned and carried out the simulations. O A, S H, M SH, H B, and P A contributed to sample preparation. O A, S H, M SH, A B, and HA N contributed to the interpretation of the results. $\mathrm{O} \mathrm{A}$ and $\mathrm{S} \mathrm{H}$ took the lead in writing the manuscript. All authors provided critical feedback and helped shape the research, analysis, and manuscript. The authors read and approved the final manuscript.

Funding

Not applicable.

Availability of data and materials

The datasets used and/or analyzed during the current study available from the corresponding author on reasonable request.

\section{Declarations}

Ethics approval and consent to participate

This study was reviewed by the Ethics Committee of Behbahan School of Medical Sciences. Therefore, the Ethics Committee of Behbahan Paramedical School has approved this study.

Ethics approval: IR.BHN.REC.1399.001

Because this study was a review study, there was no need for informed consent, and this was approved by the ethics committee. 


\section{Consent for publication}

Not applicable

\section{Competing interests}

We declare that we have no significant competing financial, professional, or personal interests that might have influenced the performance or presentation of the work described in this manuscript. We have described our potential competing financial, professional, and/or personal interests in the space.

\section{Author details \\ ${ }^{1}$ Gastrointestinal and Liver Diseases Research Center, Guilan University of Medical Sciences, Rasht, Iran. ²Department of Radiology Technology, Behbahan Faculty of Medical Sciences, Behbahan, Iran. ${ }^{3}$ School of Health Management \& Information Science, Iran University of Medical Science, Tehran, Iran. ${ }^{4}$ Abadan Faculty of Medical Science, Abadan, Iran. ${ }^{5}$ Department of Environmental Health Engineering, Paramedical School, Behbahan Faculty of Medical Sciences, Behbahan, Iran. ${ }^{6}$ Social Determinants of Health Research Center, Health Research Institute, Babol University of Medical Sciences, Babol, Iran. ${ }^{7}$ Behbahan Faculty of Medical Sciences, Behbahan, Iran.}

\section{Received: 9 January 2021 Accepted: 7 March 2021}

Published online: 25 March 2021

\section{References}

1. Chan-Yeung M, Xu R (2003) SARS: epidemiology. Respirology 8:9-14

2. Ramadan N, Shaib H (2019) Middle East respiratory syndrome coronavirus (MERS-CoV): A review. Germs. 9(1):35

3. Commission WMH. Report of clustering pneumonia of unknown etiology in Wuhan City. Wuhan Municipal Health Commission: Wuhan City health committee. 2019

4. Organization WH. Novel coronavirus ( 2019-nCoV): situation report, 3. 2020

5. Wang W, Tang J, Wei F (2020) Updated understanding of the outbreak of 2019 novel coronavirus (2019-nCoV) in Wuhan, China. J Med Virol 92(4):4417

6. Corman VM, Albarrak AM, Omrani AS, Albarrak MM, Farah ME, Almasri M, et al (2016) Viral shedding and antibody response in 37 patients with Middle East respiratory syndrome coronavirus infection. Clin Infect Dis 62(4):477-83

7. Lei J, Li J, Li X, Qi X (2020) CT imaging of the 2019 novel coronavirus (2019nCoV) pneumonia. Radiology 295(1):18

8. Fang $Y$, Zhang H, Xie J, Lin M, Ying L, Pang P, et al (2020) Sensitivity of chest CT for COVID-19: comparison to RT-PCR. Radiology 296(2):115-7

9. Ai T, Yang Z, Hou H, Zhan C, Chen C, Lv W, et al (2020) Correlation of chest CT and RT-PCR testing for coronavirus disease 2019 (COVID-19) in China: a report of 1014 cases. Radiology 296(2):32-40

10. Xie X, Zhong Z, Zhao W, Zheng C, Wang F, Liu J (2020) Chest CT for typical coronavirus disease 2019 (COVID-19) pneumonia: relationship to negative RT-PCR testing. Radiology 296(2):41-5

11. Kanne JP, Little BP, Chung JH, Elicker BM, Ketai LH (2020) Essentials for radiologists on COVID-19: an update-radiology scientific expert panel. Radiology (1):1-2

12. Xia W, Shao J, Guo Y, Peng X, Li Z, Hu D (2020) Clinical and CT features in pediatric patients with COVID-19 infection: different points from adults. Pediatr Pulmonol 55(5):1169-74

13. Li W, Cui H, Li K, Fang Y, Li S (2020) Chest computed tomography in children with COVID-19 respiratory infection. Pediatr Radiol 1(1):1-4

14. Xu X, Yu C, Qu J, Zhang L, Jiang S, Huang D, et al (2020) Imaging and clinical features of patients with 2019 novel coronavirus SARS-CoV-2. Eur J Nucl Med Mol Imaging 47(5):1275-80

15. Yang W, Cao Q, Qin L, Wang X, Cheng Z, Pan A, et al (2020) Clinical characteristics and imaging manifestations of the 2019 novel coronavirus disease (COVID-19): a multi-center study in Wenzhou city, Zhejiang, China. J Infect 80(4):388-93

16. Xu Y-H, Dong J-H, An W-M, Lv X-Y, Yin X-P, Zhang J-Z, et al (2020) Clinical and computed tomographic imaging features of novel coronavirus pneumonia caused by SARS-CoV-2. J Infect 80(4):394-400

17. Shi H, Han X, Jiang N, Cao Y, Alwalid O, Gu J, et al (2020) Radiological findings from 81 patients with COVID-19 pneumonia in Wuhan, China: a descriptive study. Lancet Infect Dis 20(4):425-34
18. Chung M, Bernheim A, Mei X, Zhang N, Huang M, Zeng X, et al (2020) CT imaging features of 2019 novel coronavirus (2019-nCoV). Radiology 295(1): 202-7

19. Fang $Y$, Zhang $H, X u Y$, Xie J, Pang $P$, Ji W (2020) CT manifestations of two cases of 2019 novel coronavirus (2019-nCoV) pneumonia. Radiology 295(1): 208-9

20. Bernheim A, Mei X, Huang M, Yang Y, Fayad ZA, Zhang N, et al (2020) Chest CT findings in coronavirus disease-19 (COVID-19): relationship to duration of infection. Radiology 1(1):42-48

21. Bai HX, Hsieh B, Xiong Z, Halsey K, Choi JW, Tran TML, et al (2020) Performance of radiologists in differentiating COVID-19 from non-COVID-19 viral pneumonia at chest CT. Radiology 296(2):46-54

22. Dai W-c, Zhang H-w, Yu J, Xu H-j, Chen H, Luo S-p, et al (2020) CT imaging and differential diagnosis of COVID-19. Can Assoc Radiol J 71(2):195-200

23. Li Y, Xia L (2020) Coronavirus disease 2019 (COVID-19): role of chest CT in diagnosis and management. Am J Roentgenol 214(6):1280-6

24. Zhou S, Wang Y, Zhu T, Xia L (2020) CT features of coronavirus disease 2019 (COVID-19) pneumonia in 62 patients in Wuhan, China. Am J Roentgenol 214(6):1287-94

25. Zhao W, Zhong Z, Xie X, Yu Q, Liu J (2020) Relation between chest CT findings and clinical conditions of coronavirus disease (COVID-19) pneumonia: a multicenter study. Am J Roentgenol 214(5):1072-7

26. Wu J, Wu X, Zeng W, Guo D, Fang Z, Chen L, et al (2020) Chest CT findings in patients with coronavirus disease 2019 and its relationship with clinical features. Investig Radiol 55(5):257

27. Albarello F, Pianura E, Di Stefano F, Cristofaro M, Petrone A, Marchioni L, et al (2020) 2019-novel coronavirus severe adult respiratory distress syndrome in two cases in Italy: an uncommon radiological presentation. Int Infect Dis 93:192-7

28. Yoon SH, Lee KH, Kim JY, Lee YK, Ko H, Kim KH, et al (2020) Chest radiographic and CT findings of the 2019 novel coronavirus disease (COVID19): analysis of nine patients treated in Korea. Korean J Radiol 21(4):494

29. Li K, Wu J, Wu F, Guo D, Chen L, Fang Z, et al (2020) The clinical and chest ct features associated with severe and critical COVID-19 pneumonia. Investig Radiol 1(1):22-24

30. Kanne JP (2020) Chest CT findings in 2019 novel coronavirus (2019-nCoV) infections from Wuhan, China: key points for the radiologist. Radiological Society of North America 2(1):45-50

31. Al-Tawfiq JA, Memish ZA (2020) Diagnosis of SARS-CoV-2 infection based on CT scan vs RT-PCR: reflecting on experience from MERS-CoV. J Hosp Infect 105(2):154-5

32. An P, Song P, Lian K, Wang Y (2020) CT Manifestations of novel coronavirus pneumonia: a case report. Balkan Med J 37(3):163

33. Bischof $\mathrm{E}_{1}$ Chen $\mathrm{G}$, Ferretti MT (2020) Understanding COVID-19 new diagnostic guidelines-a message of reassurance from an internal medicine doctor in Shanghai. Swiss Med Wkly 150(9):10

34. Chen R, Chen J, Meng Q-t (2020) Chest computed tomography images of early coronavirus disease (COVID-19). Can J Anesth 67(6):754-5

35. Chen Z-M, Fu J-F, Shu Q, Chen Y-H, Hua C-Z, Li F-B, et al (2020) Diagnosis and treatment recommendations for pediatric respiratory infection caused by the 2019 novel coronavirus. World J Pediatr 16(3):240-6

36. Duan Y-n, Qin J (2020) Pre-and posttreatment chest CT findings: 2019 novel coronavirus (2019-nCoV) pneumonia. Radiology 295(1):21

37. Huang P, Liu T, Huang L, Liu H, Lei M, Xu W, et al (2020) Use of chest CT in combination with negative RT-PCR assay for the 2019 novel coronavirus but high clinical suspicion. Radiology 295(1):22-3

38. Kanne JP (2020) Chest CT findings in 2019 novel coronavirus (2019-nCoV) infections from Wuhan, China: key points for the radiologist. Radiology 3(1):22-26

39. Kim H (2020) Outbreak of novel coronavirus (COVID-19): what is the role of radiologists? Springer 1(1):3266-3267

40. Lee EY NM-Y, Khong P-L (2020) COVID-19 pneumonia: what has CT taught us?. Lancet Infect Dis 20(4):384-385

41. Lee KS (2020) Pneumonia associated with 2019 novel coronavirus: can computed tomographic findings help predict the prognosis of the disease? Korean J Radiol 21(3):257-258. https://doi.org/10.3348/kjr.2020.0096

42. Li X, Qian Y, Liu B, Yu Y (2020) Helping the radiologist: the role of scientific journals to help prevent the spread of COVID-19. Radiology 3(1):4

\section{Publisher's Note}

Springer Nature remains neutral with regard to jurisdictional claims in published maps and institutional affiliations. 